https://doi.org/10.36785/BUITEMS.JAES.279

\title{
Physical Assessment of Marine Debris Along the Coast of Brunei Darussalam
}

\author{
Zahid Naeem Qaisrani a,b,c*, S. Shams ${ }^{\mathrm{a}}$, Guo Zhenren ${ }^{\mathrm{a}}$, Asadullah ${ }^{\mathrm{b}}$, Kuaanan Techato ${ }^{\mathrm{c}}$ \\ ${ }^{a}$ Civil Engineering Program Area, Universiti Teknologi Brunei (UTB), Jalan Tungku Link, Gadong, \\ BE 1410, Brunei Darussalam \\ ${ }^{\mathrm{b}}$ Department of Chemical Engineering, Balochistan University of Information Technology, \\ Engineering and Management Sciences (BUITEMS), Airport Road Quetta, 87300, Pakistan \\ ${ }^{\mathrm{c}}$ Faculty of Environmental Management, Prince of Songkla University (PSU), Hatyai, 90110, \\ Thailand \\ *Corresponding Author: engr.zbaloch@gmail.com
}

\begin{abstract}
Debris along coastlines is a global issue as it affects ecosystem, human health, tourism and economy; thus, requires more attention from town planners, policy makers and researchers. Various studies have been conducted around the world to identify and quantify the debris, its sources and mitigation strategies; however, it is a pioneer study of its kind in Brunei Darussalam. The current study involves selection of different beaches, debris collection and its physical analysis. Brunei Darussalam has $161 \mathrm{~km}$ long coast along South China Sea and the debris was collected from four different beaches in the month of May considering different sources related to anthropogenic, riverine and seabased activities. The selected areas for study were $110 \times 30 \mathrm{~m}^{2}$ and collected samples were categorized by number, weight, size and colour. By number, large amount of plastic $(91.46 \%)$ was found on all four beaches followed by miscellaneous materials. As, the most abundant type of debris was plastic, hence it was further classified on the basis of size and colour. Most of the materials found on these beaches were the result from land based human activities, but the contribution of debris through the waterways is also significant.
\end{abstract}

Key words-- Brunei Darussalam; Debris classification; Marine debris; Plastic pollution; Sea beaches

Date Received 11-07-2019

Date Accepted 25-12-2019

Date Published 31-12-2019

\section{INTRODUCTION}

Marine debris is "any persistent solid material that is manufactured or processed and directly or indirectly, intentionally or unintentionally, disposed of or abandoned into the marine environment of great lakes" [1]. Mainly plastic waste is found in coastal zones all over the world [2]. It adversely effects marine environment and human life[3]. In another report it is stated that more than $90 \%$ of litter on beaches is plastic [4]. Urbanization and rapid industrialization along the coastal areas make the situation more worse in many areas of the world [5]. Initially no serious attention was paid on negative impacts of plastic material [6]; however it became a serious problem as more research was carried out on its potential health impacts both on aquatic and human lives directly or indirectly[7] [8] [9]. Many factors including low cost, durability, light weight, easy availability and others made plastic a popular and extensively used product. With increase in population and their growing demands, the quantity of plastic material is increased rapidly with time [10].The annual production of plastic has been increased 200-fold from 1950 to 2014 [11]. Another research reports that during 2010, 4.8 to
12.7 million metric tons of plastic reached the oceans around the globe [12]. Some researchers described that about 5.25 trillion plastic particles are floating in world's oceans/water [12]. The floating debris not only represents unpleasant views but also have significant adverse impact on ecosystem, human health, tourism and economy[13] [14] [15] [16]. More or less all marine environment users are affected by debris to some extent including boats, divers, ship propellers and many more. Entanglement and ingestion by animals and birds are very common consequences observed all over the world [11]. Entanglement may cause serious issues for sea animals (sea turtles, fishes, birds, mammals), divers, choking of impellers of ships and many more [17]. Aquatic animals ingest this product as food by mistake where suffocation and internal injuries are resulted [18]. Sea turtles are affected particularly as they consider floating and light synthetic material as jellyfish for their food [19]. Also sometimes different harmful chemicals attached with these plastics or added during manufacturing of this polymeric material become the part of marine debris [20]. Moreover the plastic material creates waste disposal problem due to its durable characteristics [21]. Various synthetic materials found on beaches 
are possibly result of human activities. Although many studies exist on marine litter [22] [23]; however some areas are still not considered for such studies including Brunei Darussalam. This is one of the pioneer studies of its kind which analyses the debris on four selected beaches of Brunei Darussalam. Brunei Darussalam has a unique attraction for tourists in the region due to its historical places such as "Kampong Ayer" (water villages) and "Istana Nurul Iman Palace" (Palace of the light of Faith). Chen reported that Kampong Ayer is about 1000 years old and was named as "Venice of the East" by Antonio Pigafetta in 1521 [24]. Marine debris is one of the growing environmental issues in Brunei Darussalam like many other parts of the world including direct and indirect littering. Hence, tourism activities may put extensive pressure on these beaches which is an alarming situation especially in context of 2035 clean environment vision of Brunei Darussalam [25]. Therefore, the study is an effort to identify and analyses marine debris in Brunei Darussalam, as it can influence the water quality in tidal rivers and thereby put pressure on aquatic lives and mangrove ecosystem for which Brunei Darussalam is well known. The current study is unique as it provides a baseline for further research in this area, as studied sites cover direct tourism based and riverine based littering on beaches. Different methods exist for surveying, however marine debris is generally handled by land-based surveys [26] [25] [27] [23]. Samples were collected in May 2016 for this study. Based on land and riverine impacts, the study sites were classified into two groups namely A and B. Two beaches (Muara and Tungku) with land-based effects are in group A while Lumut and Seri Kenangan beaches with riverine impacts are put in group B. i.e. the latter two beaches are linked to rivers. Samples were segregated on the basis of different criteria such as number, weight, size, colour [28]. Non-biodegradable and the most abundant materials "plastic" was then focused in current study.

\section{MATERIALS AND METHODS}

\section{A. Study Area and Analysis}

$161 \mathrm{~km}$ long coastline along South China Sea, Brunei has $100 \%$ coastal population (within $100 \mathrm{~km}$ of coast) whereas its total population was about 4,23,000 people [29]. Besides tourism and recreational activities; there are commercial and residential activities which contribute in littering. Brunei has attractive and very nice sandy beaches which attract more people. More people are observed on Muara and Tungku beaches especially during weekends. The hotspots were considered between coordinates $05^{\circ} 02.284 \mathrm{~N}, 115^{\circ} 04.648 \mathrm{E}$, $05^{\circ} 02.298 \mathrm{~N}, \quad 115^{\circ} 04.648 \mathrm{E}, \quad 05^{\circ} 02.278 \mathrm{~N}, 115^{\circ}$ $04.586 \mathrm{E}$ and $05^{\circ} 02.294 \mathrm{~N}, 115^{\circ} 04.589 \mathrm{E}$ for Muara beach, coordinates $04^{\circ} 58.376 \mathrm{~N}, 114^{\circ} 52.374 \mathrm{E}$, $04^{\circ} 58.362 \mathrm{~N}, 114^{\circ} 52.383 \mathrm{E}, 04^{\circ} 58.341 \mathrm{~N}, 114^{\circ}$
52.324E and $04^{\circ} 58.358 \mathrm{~N}, 114^{\circ} 52.324 \mathrm{E}$ for Tungku beach, coordinates $4^{\circ} 40.211 \mathrm{~N}, 114^{\circ} 27.364 \mathrm{E}$, $4^{\circ} 40.187 \mathrm{~N}, \quad 114^{\circ} 27.312 \mathrm{E}, \quad 4^{\circ} 40.227 \mathrm{~N}, \quad 114^{\circ}$ $27.394 \mathrm{E}$ and $4^{\circ} 40.199 \mathrm{~N}, 114^{\circ} 27.364 \mathrm{E}$ for Lumut beach, and coordinates $04^{\circ} 08.171 \mathrm{~N}, 114^{\circ} 37.922 \mathrm{E}$, $04^{\circ} 08.185 \mathrm{~N}, 114^{\circ} 37.912 \mathrm{E}, 04^{\circ} 08.145 \mathrm{~N}, 114^{\circ}$ $37.945 \mathrm{E}$ and $04^{\circ} 08.145 \mathrm{~N}, 114^{\circ} 37.869 \mathrm{E}$ for Seri Kenangan beach. Each site for group A was 110x30 $\mathrm{m} 2$ while $(110 \times 25 \mathrm{~m} 2)$ and $(110 \times 27 \mathrm{~m} 2)$ for Lumut and Seri Kenangan beaches in group B. The difference in width is due to narrow location of study sites. Study areas are shown in Figure 1. Samples were washed to remove attached material including sand, salt etc and dried well in oven at $40^{\circ} \mathrm{C}$.

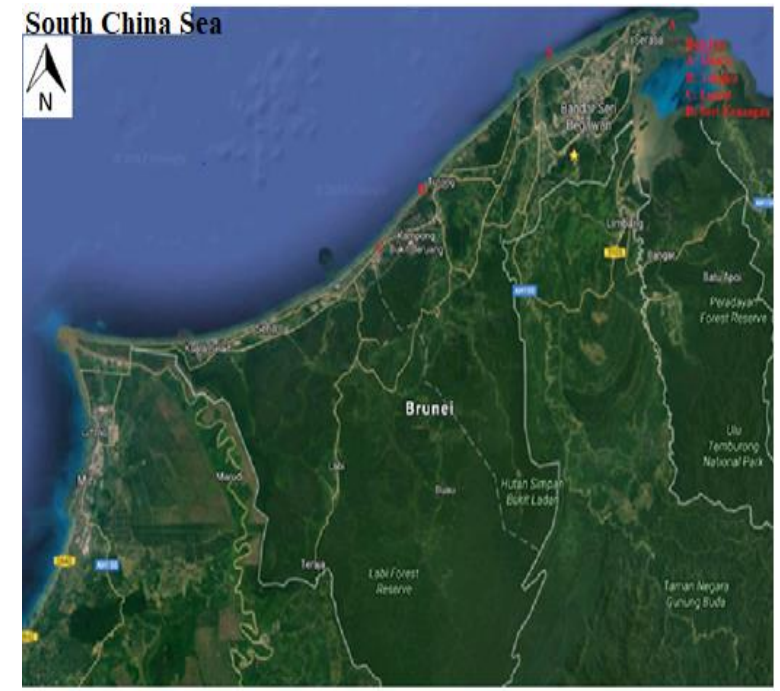

Figure 1 The selected beaches along Brunei coast (A: Muara (M); B: Tungku (T); C: Lumut (L) and D: Seri Kenangan (SK))

\section{B. Sampling}

The month of May was selected for study to check the effect of rivers as this season is wet in Brunei. These sites are open for public, commercial and recreational activities.
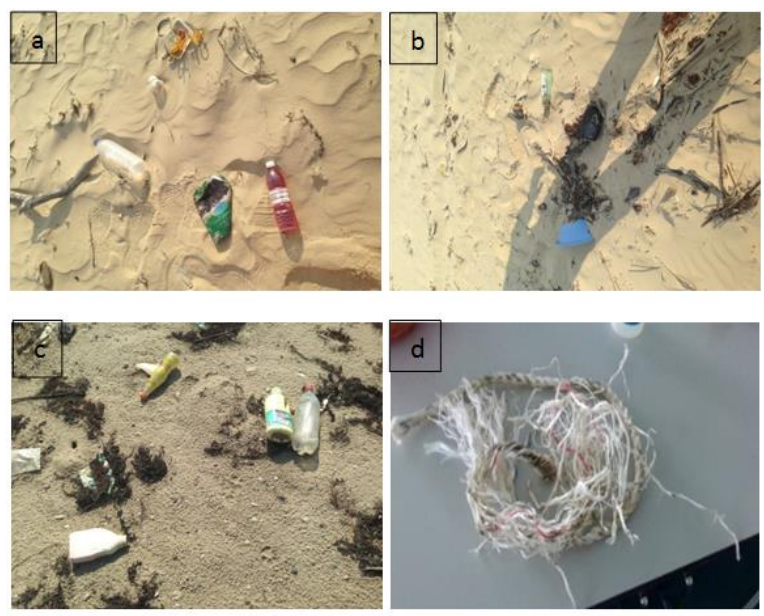

Figure 2 Representative samples collected from different beaches 
Before $1^{\text {st }}$ day of sampling, the area selected and cleaned for visible debris. The visible debris from all beaches was collected. Few representative samples are shown in Figure 2 (a, b, c and d).

\subsection{Classification}

The debris collected, washed and classified into seven main categories namely plastic, metal, rubber, glass, wood, cloth/fibre and miscellaneous [30]. Due to its abundance and non-biodegradable nature; plastic was further classified based on size and colour. The concentration of debris was calculated as;

$\mathrm{C}=\mathrm{n} /(\mathrm{w} * \mathrm{l})$

Where;

$\mathrm{C}=$ Concentration of debris (Number/square meter) $\mathrm{n}=$ No. of macro-items observed $\mathrm{w}=$ Width of the sampling site

Table 1,

Table 2, Table 3 and

Table 4.
$1=$ Length of sampling site

\section{RESULTS}

A. Quantities of Debris

By following the method prescribed by Lippiatt, the collected marine debris during May 2016 on daily basis for seven days from each site was classified into 7 major types [30]. The total number of collected items was 2050 items having weight of $176.09 \mathrm{~kg}$ shown in

Figure 3. The average number of items (weight) were $84.57(18.73 \mathrm{~kg}), 75.14(2.62 \mathrm{~kg}), 56(2.21 \mathrm{~kg})$ and $77.14(1.59 \mathrm{~kg})$ counts/day (weight, $\mathrm{kg} /$ day) on Muara, Tungku, Lumut and Seri Kenangan respectively shown in

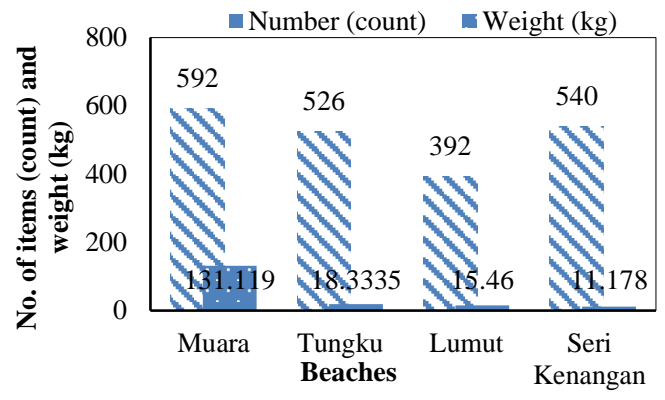

Figure 3 Number and weight of debris collected from four beaches

Also, abundance $(\%)$ by number and weight on all study sites can be seen in Figure 4 and Figure 5 respectively.

Table 1 Abundance of debris (A, number of items; B, weight in kg) at Muara beach. (May 6-12, 2016)

\begin{tabular}{|c|c|c|c|c|c|c|c|c|c|}
\hline \multirow{2}{*}{ S\# } & \multirow{2}{*}{ Day } & \multicolumn{7}{|c|}{ Material } & \multirow{2}{*}{$\begin{array}{l}\text { Total } \\
\text { (No.) }\end{array}$} \\
\hline & & Plastic & Metal & Glass & Rubber & Cloth & Lumber & Miscellaneous & \\
\hline 1 & Fri & 73 & 0 & 2 & 0 & 0 & 2 & 3 & 80 \\
\hline 2 & Sat & 124 & 5 & 0 & 0 & 1 & 1 & 6 & 137 \\
\hline 3 & Sun & 76 & 0 & 0 & 0 & 0 & 1 & 5 & 82 \\
\hline 4 & Mon & 102 & 2 & 0 & 1 & 2 & 5 & 6 & 118 \\
\hline 5 & Tue & 79 & 1 & 1 & 0 & 0 & 1 & 6 & 88 \\
\hline 6 & Wed & 18 & 2 & 0 & 0 & 1 & 0 & 0 & 21 \\
\hline 7 & Thu & 49 & 5 & 2 & 0 & 0 & 8 & 2 & 66 \\
\hline \multicolumn{2}{|c|}{ Total } & 521 & 15 & 5 & 1 & 4 & 18 & 28 & 592 \\
\hline \multicolumn{2}{|c|}{ Mean value } & 74.428 & 2.143 & 0.714 & 0.143 & 0.571 & 2.571 & 4 & 84.571 \\
\hline \multicolumn{2}{|c|}{ Std. deviation } & 34.33 & 2.115 & 0.951 & 0.378 & 0.786 & 2.878 & 2.380 & 37.175 \\
\hline
\end{tabular}

\begin{tabular}{|c|c|c|c|c|c|c|c|c|c|}
\hline \multirow{2}{*}{ S\# } & \multirow{2}{*}{ Day } & \multicolumn{7}{|c|}{ Material } & \multirow{2}{*}{$\begin{array}{c}\text { Total } \\
(\mathrm{kg})\end{array}$} \\
\hline & & Plastic & Metal & Glass & Rubber & Cloth & Lumber & Miscellaneous & \\
\hline 1 & Fri & 1.837 & 0 & 0.68 & 0 & 0 & 0.06 & 0.05 & 2.627 \\
\hline 2 & Sat & 5.335 & 3.955 & 0 & 0 & 0.005 & 0.015 & 0.07 & 9.38 \\
\hline
\end{tabular}




\begin{tabular}{|l|c|c|c|c|c|c|c|c|c|}
\hline 3 & Sun & 5.976 & 0 & 0 & 0 & 0 & 0.01 & 0.10 & 6.085 \\
\hline 4 & Mon & 4.835 & 2.94 & 0 & 0.005 & 0.09 & 0.28 & 0.18 & 8.33 \\
\hline 5 & Tue & 8.93 & 0.075 & 0.22 & 0 & 0 & 0.015 & 0.185 & 9.425 \\
\hline 6 & Wed & 7.065 & 1.005 & 0 & 0 & 0.013 & 0 & 0 & 8.083 \\
\hline 7 & Thu & 2.684 & 64.685 & 0.55 & 0 & 0 & 14.02 & 5.25 & 87.189 \\
\hline \multicolumn{2}{|c|}{ Total } & 36.661 & 72.66 & 1.45 & 0.005 & 0.108 & 14.40 & 5.835 & 131.119 \\
\hline \multicolumn{2}{|c|}{ Mean value } & 5.237 & 10.38 & 0.207 & 0.0007 & 0.015 & 2.057 & 0.833 & 18.731 \\
\hline \multicolumn{2}{r|}{ Std. deviation } & 2.441 & 23.99 & 0.292 & 0.0019 & 0.033 & 5.276 & 1.948 & 30.28 \\
\hline
\end{tabular}

Table 2 Abundance of debris (A, number of items; B, weight in kg) at Tungku beach. (May 6-12, 2016)

\begin{tabular}{|c|c|c|c|c|c|c|c|c|c|}
\hline \multirow{2}{*}{ S\# } & \multirow{2}{*}{ Day } & \multicolumn{7}{|c|}{ Material } & \multirow{2}{*}{$\begin{array}{l}\text { Total } \\
\text { (No.) }\end{array}$} \\
\hline & & Plastic & Metal & Glass & Rubber & Cloth & Lumber & Miscellaneous & \\
\hline 1 & Fri & 43 & 1 & 1 & 0 & 0 & 0 & 1 & 46 \\
\hline 2 & Sat & 96 & 2 & 0 & 0 & 2 & 4 & 3 & 107 \\
\hline 3 & Sun & 55 & 0 & 0 & 0 & 1 & 0 & 3 & 59 \\
\hline 4 & Mon & 140 & 1 & 0 & 1 & 0 & 16 & 0 & 158 \\
\hline 5 & Tue & 63 & 0 & 0 & 0 & 0 & 2 & 1 & 66 \\
\hline 6 & Wed & 35 & 0 & 0 & 0 & 0 & 0 & 3 & 38 \\
\hline 7 & Thu & 47 & 0 & 1 & 0 & 0 & 2 & 2 & 52 \\
\hline \multicolumn{2}{|c|}{ Total } & 479 & 4 & 2 & 1 & 3 & 24 & 13 & 526 \\
\hline \multicolumn{2}{|c|}{ Mean value } & 68.428 & 0.571 & 0.286 & 0.143 & 0.428 & 3.428 & 1.857 & 75.143 \\
\hline \multicolumn{2}{|c|}{ Std. deviation } & 37.228 & 0.787 & 0.488 & 0.378 & 0.787 & 5.740 & 1.215 & 42.795 \\
\hline
\end{tabular}

B. Weight of items in $\mathrm{kg}$

\begin{tabular}{|c|c|c|c|c|c|c|c|c|c|}
\hline \multirow{2}{*}{ S\# } & \multirow{2}{*}{ Day } & \multicolumn{7}{|c|}{ Material } & \multirow{2}{*}{ Total (kg) } \\
\hline & & Plastic & Metal & Glass & Rubber & Cloth & Lumber & Miscellaneous & \\
\hline 1 & Fri & 3.1 & 0.1 & 0.115 & 0 & 0 & 0 & 0.05 & 3.365 \\
\hline 2 & Sat & 1.745 & 0.105 & 0 & 0 & 0.085 & 0.03 & 2.365 & 4.33 \\
\hline 3 & Sun & 1 & 0 & 0 & 0 & 0.015 & 0 & 0.005 & 1.02 \\
\hline 4 & Mon & 1.2275 & 0.05 & 0 & 0.01 & 0 & 0.17 & 0 & 1.4575 \\
\hline 5 & Tue & 1.16 & 0 & 0 & 0 & 0 & 0.45 & 0.005 & 1.615 \\
\hline 6 & Wed & 3.89 & 0 & 0 & 0 & 0 & 0 & 0.105 & 3.995 \\
\hline 7 & Thu & 2.261 & 0 & 0.175 & 0 & 0 & 0.045 & 0.07 & 2.551 \\
\hline \multicolumn{2}{|c|}{ Total } & 14.3835 & 0.255 & 0.29 & 0.01 & 0.1 & 0.695 & 2.6 & 18.3335 \\
\hline \multicolumn{2}{|c|}{ Mean value } & 2.055 & 0.036 & 0.041 & 0.00143 & 0.014 & 0.099 & 0.371 & 2.619 \\
\hline \multicolumn{2}{|c|}{ Std. deviation } & 1.095 & 0.04 & 0.07 & 0.00378 & 0.03 & 0.166 & 0.88 & 1.310 \\
\hline
\end{tabular}

Table 3 Abundance of debris (A, number of items; B, weight in kg) at Lumut beach. (May 20-26, 2016)

\begin{tabular}{|c|c|c|c|c|c|c|c|c|}
\hline \multirow{2}{*}{ S\# } & \multirow{2}{*}{ Day } & \multicolumn{9}{|c|}{ Material } & Lumber & Miscellaneous & Total \\
\cline { 2 - 9 } & & Plastic & Metal & Glass & Rubber & Cloth & Lumber \\
\hline 1 & Fri & 61 & 0 & 0 & 0 & 1 & 1 & 5 \\
\hline
\end{tabular}




\begin{tabular}{|l|c|c|c|c|c|c|c|c|c|}
\hline 2 & Sat & 55 & 0 & 0 & 0 & 0 & 0 & 57 \\
\hline 3 & Sun & 59 & 1 & 0 & 2 & 0 & 1 & 0 \\
\hline 4 & Mon & 72 & 0 & 0 & 1 & 1 & 0 & 1 \\
\hline 5 & Tue & 72 & 0 & 0 & 0 & 0 & 1 & 0 \\
\hline 6 & Wed & 39 & 0 & 0 & 1 & 0 & 1 & 0 & 0 \\
\hline 7 & Thu & 13 & 0 & 0 & 0 & 0 & 43 & 0 \\
\hline & Total & 371 & 1 & 0 & 4 & 2 & 4 & 13 \\
\hline & Mean value & 53 & 0.143 & 0 & 0.571 & 0.286 & 0.571 & 1.428 \\
\hline
\end{tabular}

B. Weight of items $(\mathrm{kg})$

\begin{tabular}{|c|c|c|c|c|c|c|c|c|c|}
\hline \multirow{2}{*}{ S\# } & \multirow{2}{*}{ Day } & \multicolumn{9}{|c|}{ Material } & Total \\
\cline { 3 - 10 } & & Plastic & Metal & Glass & Rubber & Cloth & Lumber & Miscellaneous & \\
\hline 1 & Fri & 0.605 & 0 & 0 & 0 & 0.265 & 0.02 & 0.19 & 1.08 \\
\hline 2 & Sat & 1.78 & 0 & 0 & 0 & 0 & 0 & 0.07 & 1.85 \\
\hline 3 & Sun & 1.28 & 0.22 & 0 & 0.005 & 0 & 0.025 & 0.145 & 1.675 \\
\hline 4 & Mon & 0.615 & 0 & 0 & 0.005 & 0.05 & 0 & 0.03 & 0.7 \\
\hline 5 & Tue & 0.615 & 0 & 0 & 0 & 0 & 0.005 & 0 & 0.62 \\
\hline 6 & Wed & 0.155 & 0 & 0 & 9.255 & 0 & 0.01 & 0 & 9.42 \\
\hline 7 & Thu & 0.115 & 0 & 0 & 0 & 0 & 0 & 0 & 0.115 \\
\hline \multicolumn{2}{|c|}{ Total } & 5.165 & 0.22 & 0 & 9.265 & 0.315 & 0.06 & 0.435 & 15.46 \\
\hline \multicolumn{2}{|c|}{ Mean value } & 0.738 & 0.03 & 0 & 1.323 & 0.045 & 0.008 & 0.062 & 2.208 \\
\hline \multicolumn{2}{|c|}{ Std. deviation } & 0.599 & 0.08 & 0 & 3.497 & 0.988 & 0.010 & 0.077 & 3.237 \\
\hline
\end{tabular}

Table 4 Abundance of debris (A, number of items; B, weight in kg) at Seri Kenangan beach (May 20-26, 2016)

\begin{tabular}{|c|c|c|c|c|c|c|c|c|c|}
\hline \multirow{2}{*}{$\begin{array}{c}\text { S } \\
\#\end{array}$} & Day & \multicolumn{9}{|c|}{ Material } & Total \\
\hline 1 & & Plastic & Metal & Glass & Rubber & Cloth & Lumber & Miscellaneous & \\
\hline 2 & Fri & 176 & 0 & 0 & 2 & 0 & 7 & 8 & 193 \\
\hline 3 & Sun & 76 & 0 & 1 & 0 & 0 & 2 & 4 & 83 \\
\hline 4 & Mon & 50 & 0 & 0 & 0 & 0 & 1 & 0 & 103 \\
\hline 5 & Tue & 45 & 0 & 1 & 2 & 0 & 1 & 1 & 51 \\
\hline 6 & Wed & 49 & 0 & 0 & 0 & 0 & 1 & 1 & 51 \\
\hline 7 & Thu & 9 & 0 & 0 & 0 & 0 & 0 & 0 & 9 \\
\hline \multicolumn{2}{|c|}{ Total } & 504 & 1 & 2 & 5 & 0 & 13 & 15 & 540 \\
\hline \multicolumn{2}{|c|}{ Mean value } & 72 & 0.143 & 0.286 & 0.714 & 0 & 1.857 & 2.143 & 77.143 \\
\hline \multicolumn{2}{|c|}{ Std. deviation } & 53.653 & 0.378 & 0.488 & 0.951 & 0 & 2.34 & 2.911 & 58.98 \\
\hline
\end{tabular}

B. Weight of items in $\mathrm{kg}$

\begin{tabular}{|c|c|c|c|c|c|c|c|c|c|}
\hline \multirow{2}{*}{ S\# } & \multirow{2}{*}{ Day } & \multicolumn{7}{|c|}{ Material } & \multirow{2}{*}{ Total } \\
\hline & & Plastic & Metal & Glass & Rubber & Cloth & Lumber & Miscellaneous & \\
\hline 1 & Fri & 3.03 & 0 & 0 & 0.015 & 0 & 0.125 & 0.09 & 3.26 \\
\hline 2 & Sat & 1.596 & 0 & 0.485 & 0 & 0 & 0.01 & 0.07 & 2.161 \\
\hline 3 & Sun & 1.64 & 0.11 & 0 & 0.09 & 0 & 0.015 & 0.015 & 1.87 \\
\hline 4 & Mon & 2.36 & 0 & 0 & 0 & 0 & 0.001 & 0 & 2.361 \\
\hline 5 & Tue & 0.67 & 0 & 0.001 & 0.005 & 0 & 0.05 & 0.03 & 0.756 \\
\hline 6 & Wed & 0.29 & 0 & 0 & 0 & 0 & 0.005 & 0.02 & 0.315 \\
\hline 7 & Thu & 0.455 & 0 & 0 & 0 & 0 & 0 & 0 & 0.455 \\
\hline \multicolumn{2}{|c|}{ Total } & 10.041 & 0.11 & 0.486 & 0.11 & 0 & 0.206 & 0.225 & 11.178 \\
\hline \multicolumn{2}{|c|}{ Mean value } & 1.434 & 0.016 & 0.069 & 0.016 & 0 & 0.029 & 0.032 & 1.597 \\
\hline \multicolumn{2}{|c|}{ Std. deviation } & 1.026 & 0.041 & 0.183 & 0.033 & 0 & 0.045 & 0.035 & 1.11 \\
\hline
\end{tabular}


By number, plastic is the most abundant part $(91.46 \%)$ followed by miscellaneous $(3.22 \%)$ while by weight, metal is the leading portion $(41.60 \%)$ followed by plastic (37.62\%) shown in Figure 4 and Figure 5. Muara and Tungku beaches were found more littered. The concentration was calculated by using NOAA method which shows 0.331028 , and 0.323751 items $/ \mathrm{m}^{2} /$ week on beaches of group A and $\mathrm{B}$ respectively shown in Table 5 separately. The density by number $\left(\mathrm{n} / \mathrm{m}^{2}\right)$ and by weight $\left(\mathrm{kg} / \mathrm{m}^{2}\right)$ per week on four mentioned beaches of Brunei is shown in Figure 6 and Figure 7 respectively.

Table 5 Density of collected debris in count $/ \mathrm{m}^{2}$ and $\mathrm{kg} / \mathrm{m}^{2}$ per week

\begin{tabular}{|c|c|c|c|c|c|c|c|c|c|}
\hline \multirow{2}{*}{ Gp } & \multirow{2}{*}{\multicolumn{2}{|c|}{ Beach }} & \multicolumn{7}{|c|}{ Material $\left\{\right.$ Count (weight) $/ \mathrm{m}^{2} /$ week $\}$} \\
\hline & & & \multirow{2}{*}{$\begin{array}{c}\text { Plastic } \\
0.158\end{array}$} & \multirow{2}{*}{$\begin{array}{c}\text { Metal } \\
0.0054\end{array}$} & \multirow{2}{*}{$\begin{array}{c}\text { Glass } \\
0.0015\end{array}$} & \multirow{2}{*}{$\begin{array}{l}\text { Rubber } \\
0.0003\end{array}$} & \multirow{2}{*}{$\begin{array}{c}\text { Cloth } \\
0.0012\end{array}$} & \multirow{2}{*}{$\begin{array}{c}\text { Lumber } \\
0.0054\end{array}$} & \multirow{2}{*}{$\begin{array}{c}\text { Miscellaneous } \\
0.0085\end{array}$} \\
\hline \multirow{4}{*}{ A } & \multirow[b]{2}{*}{ M } & Count & & & & & & & \\
\hline & & Weight & 0.011 & 0.022 & 0.00044 & $1.51 * 10^{-6}$ & $3.27 * 10^{-5}$ & $4.36^{*} 10^{-3}$ & $1.76^{*} 10^{-3}$ \\
\hline & \multirow{2}{*}{$\mathrm{T}$} & Count & 0.145 & $1.21 * 10^{-3}$ & $6.06^{*} 10^{-4}$ & $3.03 * 10^{-4}$ & $9.09 * 10^{-4}$ & $7.27 * 10^{-3}$ & $3.93 * 10^{-3}$ \\
\hline & & Weight & $4.36^{*} 10^{-3}$ & $7.73 * 10^{-5}$ & $8.79 * 10^{-5}$ & $3.03 * 10^{-6}$ & $3.03 * 10^{-5}$ & $2.11 * 10^{-4}$ & $7.88 * 10^{-4}$ \\
\hline \multirow{4}{*}{ B } & \multirow{2}{*}{$\mathrm{L}$} & Count & 0.135 & $3.64 * 10^{-4}$ & 0 & $1.45^{*} 10^{-3}$ & $7.27 * 10^{-4}$ & $1.45^{*} 10^{-3}$ & $3.64 * 10^{-3}$ \\
\hline & & Weight & $1.88 * 10^{-3}$ & $8 * 10^{-5}$ & 0 & $3.37 * 10^{-3}$ & $1.14 * 10^{-4}$ & $2.18 * 10^{-5}$ & $1.58 * 10^{-4}$ \\
\hline & \multirow{2}{*}{ SK } & Count & 0.169 & $3.37 * 10^{-4}$ & $6.73 * 10^{-4}$ & $1.68 * 10^{-3}$ & 0 & $4.38 * 10^{-3}$ & $5.05 * 10^{-3}$ \\
\hline & & Weight & $3.38 * 10^{-3}$ & $3.70 * 10^{-5}$ & $1.64 * 10^{-4}$ & $3.70^{*} 10^{-5}$ & 0 & $6.94 * 10^{-5}$ & $7.57 * 10^{-5}$ \\
\hline
\end{tabular}

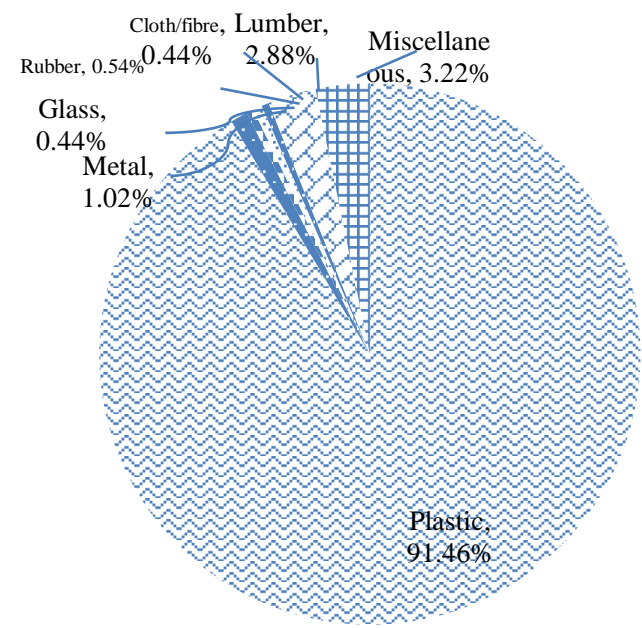

Figure 4 Abundance of different debris types by number on all four beaches

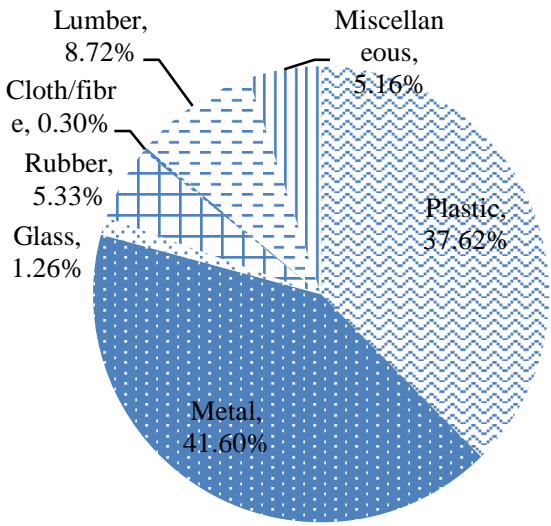

Figure 5 Abundance of different debris types by weight on all four beaches

The results of standard deviation show that plastic has the highest standard deviation while glass has the lowest; whereas, the abundance of glass, rubber and cloth/fibre have not much variation with varying sites. The mean value (average number and weight) and their respective standard deviations for each beach are also plotted in Figure 8 and Figure 9 respectively. 


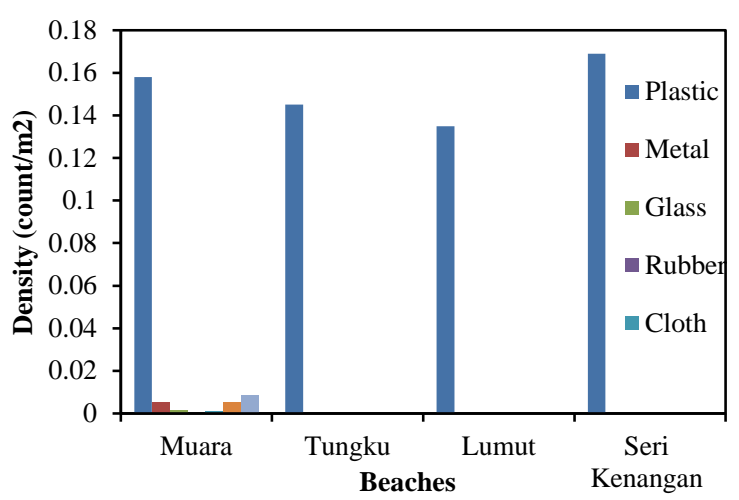

Figure 6 Density of debris on each beach $\left(\mathrm{n} / \mathrm{m}^{2}\right)$ per week

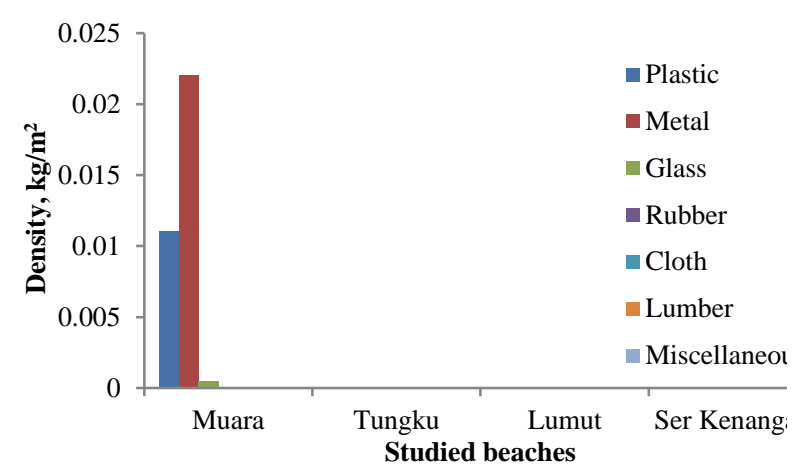

Figure 7 Density of debris by weight $\left(\mathrm{kg} / \mathrm{m}^{2}\right)$ per week

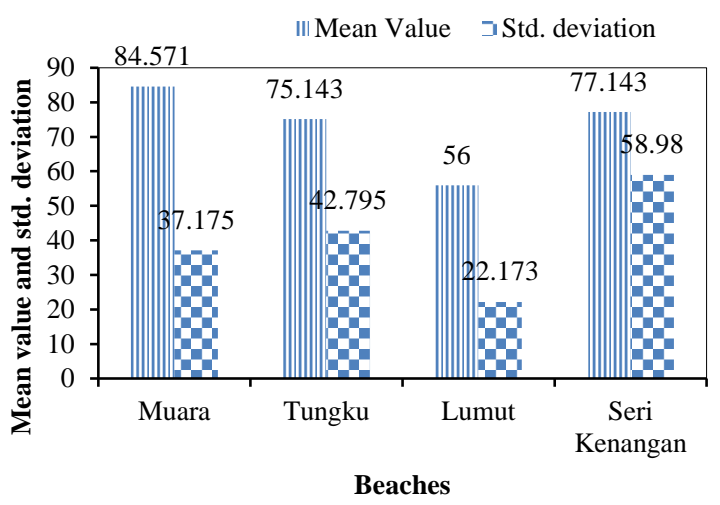

Figure 8 Mean value and standard deviation of debris by number on each beach

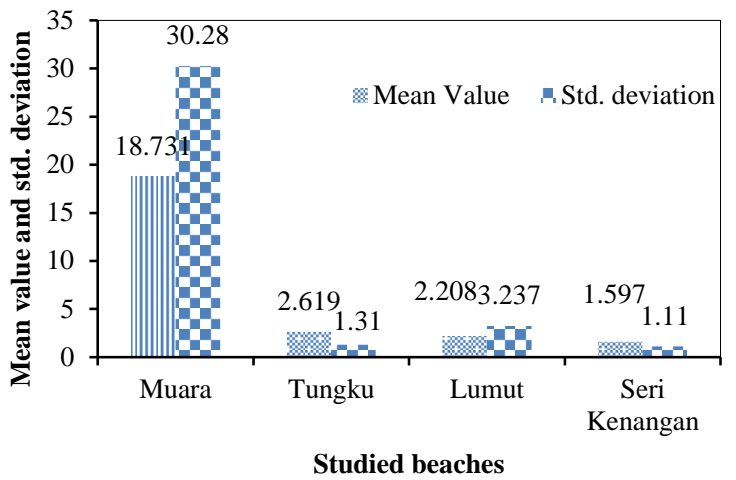

Figure 9 Mean value and standard deviation of debris by weight on

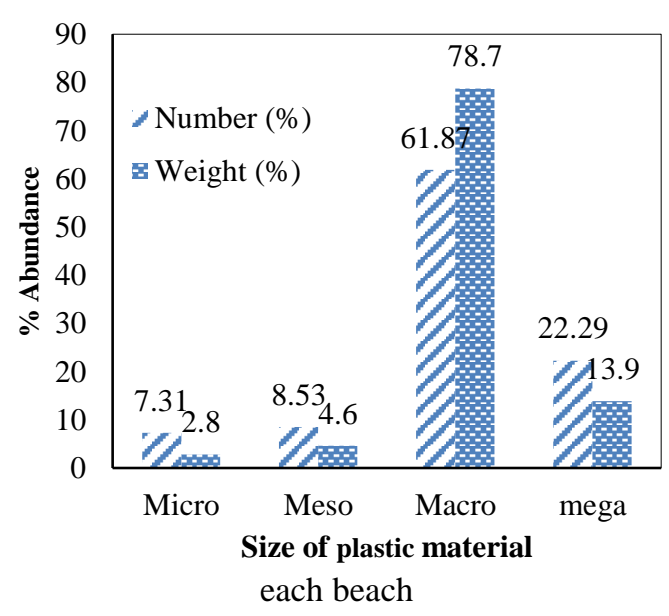

Figure $10 \%$ Abundance of plastic material on the basis of size

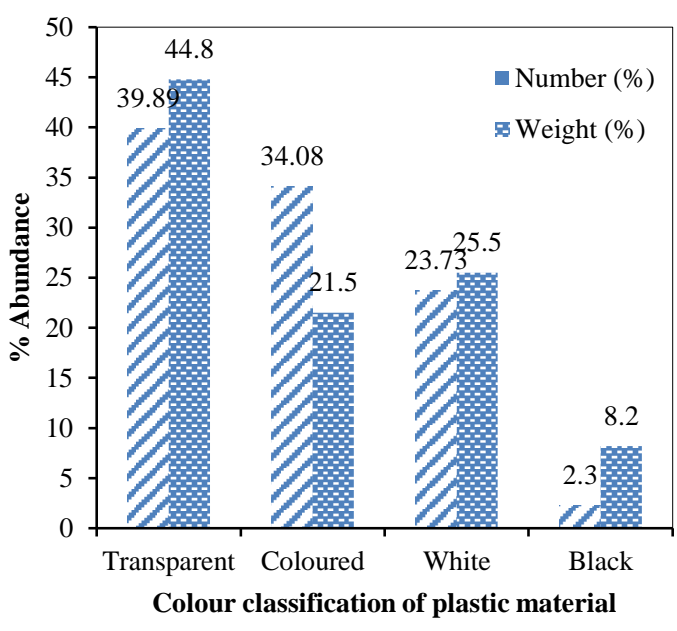

Figure $11 \%$ abundance of plastic material by colour

The plastic products were further classified by size and colour. Micro, meso, macro and mega are used for size $\leq 5 \mathrm{~mm}, 5-20 \mathrm{~mm}, 20-100 \mathrm{~mm}$ and $>100 \mathrm{~mm}$ respectively [2] [31]. Based on size and colour, 
plastic material was classified as shown in Table 6.

Table 6 Classification of plastic items by size and colour

\begin{tabular}{|c|c|c|c|c|c|c|c|c|}
\hline \multirow[b]{2}{*}{ 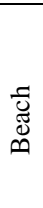 } & \multicolumn{4}{|c|}{ Size } & \multicolumn{4}{|c|}{ Colour } \\
\hline & $\stackrel{\circ}{:}$ & $\sum_{\substack{0 \\
\Sigma}}$ & 莺 & 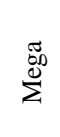 & 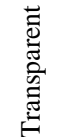 & $\begin{array}{l}\vec{D} \\
\stackrel{0}{0} \\
\dot{0}\end{array}$ & $\stackrel{\frac{g}{*}}{3}$ & $\frac{\frac{y}{\pi}}{\infty}$ \\
\hline$\Sigma$ & 10 & 22 & 334 & 148 & 204 & 169 & 127 & 15 \\
\hline 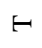 & 41 & 64 & 241 & 131 & 183 & 189 & 95 & 13 \\
\hline - & 54 & 37 & 278 & 68 & 173 & 138 & 112 & 6 \\
\hline$\frac{v}{v}$ & 32 & 37 & 307 & 71 & 188 & 143 & 111 & 9 \\
\hline$\stackrel{\pi}{0}$ & 137 & 160 & 1160 & 418 & 748 & 639 & 445 & 43 \\
\hline
\end{tabular}

Macro plastics were found abundantly (61.86\% in number and $78.7 \%$ by weight) followed by mega ( $22.29 \%$ by number and $13.9 \%$ by weight), meso ( $8.53 \%$ by number and $4.6 \%$ by weight) and micro ( $7.31 \%$ by number and $2.8 \%$ by weight); while based on colour, plastics were categorised into transparent, coloured, white and black [28]. Major part was of transparent plastics $(39.89 \%$ by number and $44.8 \%$ by weight) followed by coloured (34.08\% by number and $21.5 \%$ by weight), white ( $23.73 \%$ by number and $25.5 \%$ by weight) and black ( $2.29 \%$ by number and $8.2 \%$ by weight) shown in Figure 10 and Figure 11.

\section{B. Composition of Debris}

The plastic material was found abundantly among all found debris on these beaches. Plastic by number was $91.46 \%$ followed by miscellaneous $3.22 \%$ and lumber $2.88 \%$. By weight, metals were found abundantly $(41.59 \mathrm{~kg}$ ) followed by plastic $(37.62 \%)$, lumber $(8.72 \%)$, rubber $(5.33 \%)$, miscellaneous $(5.16 \%)$, glass $(1.26 \%)$ and cloth $(0.29 \%)$. Out of 1875 items of plastic in various forms, major contribution of food stuffs $(26.51 \%)$ followed by plastic fragments $(23.25 \%)$, PET bottles $(15.63 \%)$, polyethylene bags (13.76\%) and Polyvinyl Chloride $(1.92 \%)$ was observed as shown in Table 7.

Table 7 Major types of plastic debris

\begin{tabular}{|c|c|c|c|c|c|}
\hline \multirow{2}{*}{ Beach } & \multicolumn{5}{|c|}{ Material } \\
\cline { 2 - 6 } & FS & PF & PB & PE & PVC \\
\hline M & 121 & 146 & 123 & 36 & 4 \\
\hline T & 145 & 57 & 49 & 106 & 1 \\
\hline L & 104 & 110 & 3 & 66 & 19 \\
\hline SK & 127 & 123 & 91 & 5 & 12 \\
\hline Total & 497 & 436 & 266 & 213 & 36 \\
\hline $\begin{array}{c}\text { \% by } \\
\text { number }\end{array}$ & 34.32 & 30.11 & 18.37 & 14.70 & 2.5 \\
\hline
\end{tabular}

FS: Food Stuffs

PF: Plastic Fragments

PB: PET Bottles
PE: Poly Ethylene

PVC: Poly Vinyl chloride

\section{Sources of Debris}

Total number of items collected was counted as 2050 items with a weight of $176.09 \mathrm{Kg}$. These four beaches have been selected to measure the land based and riverine based effects on beaches for marine debris. Beaches in group A were found more littered as tourism and direct littering on these beaches are the major causes of this debris. Mostly the litter found cannot be removed completely during cleaning campaigns. Volunteer or paid campaigns only focus on large material. Although the debris collected on these beaches has some indications about the presence and contribution of neighbouring countries, but major portion is from local sources, as mostly debris found has labels of Brunei Darussalam. These results show that like other countries; Brunei Darussalam is also more affected by land-based sources. The beaches in group A (density $0.331028 \mathrm{n} / \mathrm{m}^{2} /$ week) having recreational or commercial activities were found more littered as compared to beaches in group B (density $0.323751 \mathrm{n} / \mathrm{m}^{2} /$ week).

\section{DISCUSSIONS}

As current study is physical based assessment of collected debris from four beaches of Brunei Darussalam; so, no statistical analysis was done for this work. Plastic is found the leading contributor in marine littering. Majorly types of debris and their respective quantities were focused in this work, but sources identification was of the keen interest for proper management. People behaviour towards littering on recreational sites and near waterways is found discouraging like developing countries [31]. Recreational and commercial activities make Maura beach more littered. There were clear signs of human input in littering on these beaches. As sites were not being affected by sea currents or runoff which shows the presence of local sources or landbased sources. It needs proper management and policy implementation to control this litter. Proper environmental education, provision of disposable facilities, public awareness through different means can help in litter reduction. The ban on plastic bags in Brunei Darussalam during weekends is an appreciable initiative; however, the same is being provided to customers in kitchen/small markets at the same time which needs to be considered. Hence, regular monitoring to check the provision of plastic bags to public during weekends is required. Law enforcement in the form of fines can reduce the use of plastic bags during weekends.

\section{CONCLUSIONS \& RECOMMENDATIONS}

The results show anthropogenic pressure on beaches as a result of recreational activities. Plastic products 
of various kinds are found abundant on these sites. Also, land-based sources of debris were found dominating. Continuous monitoring, systematic collection and law enforcement simultaneously can give a sustainable solution. Suitable trapping mechanisms are required to restrict the movement of debris from coast to sea. Different sources can be used for controlling plastic litter such as educating people through curriculum, public messages, TV talk shows, newspaper advertisement, displaying sign boards, public figure speeches and many more. The initial study can provide a baseline for further research. The extensive study on more beaches supported by chemical and statistical analysis can demonstrate the condition of the coastline more clearly.

\section{ACKNOWLEDGEMENT}

We are thankful to Ahmad Asyraf Bin Haji Lathif, Mohammad Nadzireen Bin Ibrahim and Mohammed Thariyqul Aqil Caesar Bin Mohammed (Ex-HND students) for helping in samples collection. Also special thanks to $\mathrm{Hj}$ Khairuldini $\mathrm{Hj}$ Metali and Alifatul Haziqah Abu Hanipah (Ph.D. students) for their valuable suggestions and encouragement during this study. Special thanks to Faculty of Environmental Management and Graduate School, Prince of Songkla University, Hatyai Campus, Thailand for provision of work station to complete this manuscript. This research did not receive any specific grant from any funding source.

\section{REFERENCES}

[1] NOAA, "Programmatic Environmental Assessment (PEA) for the NOAA Marine Debris Program (MDP)," p. $168,2013$.

J. A. Ivar do Sul, I. R. Santos, A. C. Friedrich, A. Matthiensen, and G. Fillmann, "Plastic Pollution at a Sea Turtle Conservation Area in NE Brazil: Contrasting Developed and Undeveloped Beaches," Estuaries and Coasts, vol. 34, no. 4, pp. 814-823, 2011.

[3] Mauro Mecozzi and M. Pietroletti, "Chemical composition and surfactant characteristics of marine foams investigated by means of UV-v is, FTIR and FTNIR spectroscopy," Springer, 2016.

[4] J. Sarafraz, M. Rajabizadeh, and E. Kamrani, "The preliminary assessment of abundance and composition of marine beach debris in the northern Persian Gulf, Bandar Abbas City, Iran.," J. Mar. Biol. Assoc. UK, vol. 96, pp. 131-135, 2016.

[5] M. Allsopp, A. Walters, D. Santillo, and P. JohnstonAllsopp, "Plastic debris in the world's oceans," 2006.

[6] W. C. Fergusson and J.J.P. Staudinger (Ed.), "Plastic and the environment," 1974.

[7] N. P. Purba et al., "Marine debris in Indonesia: A review of research and status," Mar. Pollut. Bull., vol. 146, no. May, pp. 134-144, 2019.

[8] J. Pinto, P. S. M. Santos, A. C. Duarte, and T. Rochasantos, "Science of the Total Environment ( Nano ) plastics in the environment - Sources, fates and effects," Sci. Total Environ., vol. 566-567, pp. 15-26, 2016.

[9] A. Stefatos, M. Charalampakis, G. Papatheodorou, and G. Ferentinos, "Marine Debris on the Sea ${ }^{-}$oor of the Mediterranean Sea: Examples from Two Enclosed Gulfs in Western Greece," vol. 36, no. 5, pp. 389-393,
1999.

[10] D.W. Laist, "Overview of the biological effects of lost and discarded plastic debris in the marine environment," Mar. Pollut. Bull., vol. 18, pp. 319-326, 1987.

[11] W. C. Li, H. F. Tse, and L. Fok, "Science of the Total Environment Plastic waste in the marine environment: A review of sources , occurrence and effects," vol. 567, pp. 333-349, 2016.

[12] O. . Hoegh-Guldberg et al., "The ocean," Clim. Chang. 2014 Impacts, Adapt. Vulnerability Part B Reg. Asp. Work. Gr. II Contrib. to Fifth Assess. Rep. Intergov. Panel Clim. Chang., no. January, pp. 1655-1734, 2015.

[13] R. H. Waring, R. M. Harris, and S. C. Mitchell, "Maturitas Plastic contamination of the food chain: A threat to human health ?," Maturitas, vol. 115, no. June, pp. 64-68, 2018.

[14] J. G. . Derraik, "The pollution of the marine environment by plastic debris: a review," Mar. Pollut. Bull., vol. 44, no. 9, pp. 842-852, 2002.

[15] M. R. Gregory, "Environmental implications of plastic debris in marine settings--entanglement, ingestion, smothering, hangers-on, hitch-hiking and alien invasions," Philos. Trans. R. Soc. Lond. B. Biol. Sci., vol. 364, no. 1526, pp. 2013-2025, 2009.

[16] C.M. Rochman, C. Manzano, and E. H. B.T. Hentschel, S.L.M. Simonich, "Polystyrene plastic: a source and sink for polycyclic aromatic hydrocarbons in the marine environment," Env. Sci Technol, vol. 47, pp. 13976-13984, 2013.

[17] H. S. Carson, "The incidence of plastic ingestion by fishes: From the prey's perspective," Mar. Pollut. Bull., vol. 74, no. 1, pp. 170-174, 2013.

[18] J. Tomás, R. Guitart, R. Mateo, and J. a Raga, "Marine debris ingestion in loggerhead sea turtles, Caretta caretta, from the Western Mediterranean.," Mar. Pollut. Bull., vol. 44, no. 3, pp. 211-216, 2002.

[19] S. C. Gall and R. C. Thompson, "The impact of debris on marine life," Mar. Pollut. Bull., vol. 92, no. 1-2, pp. 170-179, 2015.

[20] J. Gauquie, L. Devriese, J. Robbens, and B. De Witte, "A qualitative screening and quantitative measurement of organic contaminants on different types of marine plastic debris," Chemosphere, vol. 138, pp. 348-356, 2015.

[21] M. Cole, P. Lindeque, C. Halsband, and T. S. Galloway, "Microplastics as contaminants in the marine environment: A review," Mar. Pollut. Bull., vol. 62, no. 12, pp. 2588-2597, 2011

[22] I. R. Santos, A. C. Friedrich, and J. A. I. Do Sul, "Marine debris contamination along undeveloped tropical beaches from northeast Brazil," Environ. Monit. Assess., vol. 148, no. 1-4, pp. 155-462, 2009.

[23] S. Hong, J. Lee, D. Kang, H. W. Choi, and S. H. Ko, "Quantities, composition, and sources of beach debris in Korea from the results of nationwide monitoring," Mar. Pollut. Bull., vol. 84, no. 1-2, pp. 27-34, 2014.

[24] H. J. Chen, P. J. Chen, and F. Okumus, "The relationship between travel constraints and destination image: A case study of Brunei," Tour. Manag., vol. 35, pp. 198-208, 2013.

[25] Z. N. Qaisrani, S. Shams, G. Zhenren, M. S. Reza, and Q. Zaunuddin, "Quantitative analysis of marine debris along the sea beaches of Brunei Darussalam," in 7th Brunei International Conference on Engineering and Technology 2018 (BICET 2018), 2018, p. 4.

[26] C. Y. Manullang, "The abundance of Plastic Marine Debris on Beaches in Ambon Bay The abundance of Plastic Marine Debris on Beaches in," in IOP Conf. Series: Earth and Environmental Science, 2019, p. 6.

[27] Storrier, K.L., D. J. McGlashan, S. Bonellie, and K. Velander, "Beach litter deposition at a selection of beaches in the Firth of Forth, Scotland.," J. Coast. Res., vol. 23, no. 4, pp. 813-822, 2007.

[28] S. Zhao, L. Zhu, and D. Li, "Characterization of small 
plastic debris on tourism beaches around the South China Sea," Reg. Stud. Mar. Sci., vol. 1, pp. 55-62, 2015.

[29] PEMSEA, "PEMSEA (Partnerships in Environmental Management for the Seas of East Asia), 2015. Sustainable Development Strategy for the Seas of East Asia (SDS-SEA)," Quezon City, Philippines, 2015.

[30] S. Lippiatt, S. Opfer, and C. Arthur, "Marine Debris Monitoring and Assessment," NOAA Tech. Memo. NOS-OR\&R-46, no. November, 2013.

[31] H. B. Jayasiri, C. S. Purushothaman, and A. Vennila, "Plastic litter accumulation on high-water strandline of urban beaches in Mumbai, India," Environ. Monit. Assess., vol. 185, no. 9, pp. 7709-7719, 2013.

Journal of Applied and Emerging

Sciences by BUITEMS is licensed under a Creative Commons Attribution 4.0 International License. 